\title{
High Prevalence of Cardiovascular Comorbidity and Chronic Pain in Polio Survivors as Compared to the General Population
}

\author{
Schwartz I', Gartsman I', Adler B ${ }^{2}$, Friedlander \\ $\mathrm{Y}^{2}$, Manor $\mathrm{O}^{2}$, Levine $\mathrm{H}^{2}$ and Meiner $\mathrm{Z}^{1 *}$ \\ ${ }^{1}$ Department of Physical Medicine and Rehabilitation, \\ Hadassah Medical Center, Israel \\ ${ }^{2}$ The Hebrew University-Hadassah, Braun School of \\ Public Health, Israel \\ *Corresponding author: Zeev Meiner, Department \\ of Physical Medicine and Rehabilitation, POB 24035, \\ Hadassah University Hospital, J erusalem, 91240, Israel
}

Received: J anuary 20, 2018; Accepted: February 21, 2018; Published: February 28, 2018

\begin{abstract}
Objective: The aims of this study are to investigate how the interaction between polio sequelae and aging contributes to the general health and functional level of polio survivors and to identify factors which contribute to the increased morbidity of polio survivors when compared to the general population.

Design: A case-control study.

Setting: Post-polio clinic in a tertiary university center.

Participants: 195 polio patients who attended post-polio clinic.

Interventions: Not applicable.

Main Outcome Measure(s): Demographic, medical, social, and functional data was evaluated using a generalized questionnaire which was administered in a national health surveys with adaption to the polio population. This data was compared, using logistic regression analysis, to 768 age and sex matched controls from the general population.
\end{abstract}

Results: The analysis indicated that the rate of cardiovascular diseases $(p<0.0001)$, hypertension $(p=0.006)$, diabetes $(p<0.0001)$ and chronic pain $(p<0.0001)$ was significantly higher among polio patients. Male gender, hypertension and single status increased the rate of cardiovascular diseases among polio survivors. However, unlike the general population, the functional status of polio survivors was not affected by cardiovascular diseases or chronic pain.

Conclusions: Our study demonstrated that long-standing poliomyelitis has a deleterious impact on the general health and functional level of polio survivors emphasizing the need for early screening for vascular risk factors and special health promotion program to prevent their further decline.

Keywords: Poliomyelitis; Cardiovascular comorbidity; Chronic pain; Functional outcomes; Post-polio syndrome

\section{Introduction}

According to the World Health Organization it is estimated that 12-20 million people are living with sequelae of poliomyelitis worldwide [1]. Long-standing poliomyelitis has a deleterious effect on the general medical health and functional status of polio survivors as compared to the general population $[2,3]$.

During the 1950's in Israel, more than 7000 people were afflicted with paralytic poliomyelitis including a significant prevalence of childhood cases [4-6]. As a result of the illness, throughout their life span these patients suffered from various motor and functional disabilities. In a recent Israeli census conducted following the 2007 legislation of the Polio Victims Compensation Law [7], over 2500 polio survivors were identified.

Several studies have shown that paralytic polio patients are more prone than the general population to developing cardiovascular diseases (CVDs), hypertension, lung diseases, diabetes and orthopedic complications such as osteoporosis, fractures and scoliosis [8-10]. The reasons behind the increased health risks of polio survivors are unclear, though the predominant explanation is their impaired mobility and consequent sedentary lifestyle. However, these studies compared the prevalence of comorbidities alone and not factors influencing these comorbidities, such as socio-demographic information, medical risk factors and levels of disability.

The primary goals of this study were twofold; to investigate whether such disparities in the prevalence of cardiovascular comorbidities and chronic pain between polio survivors and the general population exist in Israeli polio population and if so, to identify the main factors contributing to these discrepancies.

An additional purpose of the study was to assess whether a history of polio infection and its severity are risk factors for cardiovascular disease or chronic pain. The findings of this study may be important in identifying the risk factors for medical and functional deterioration
Phys Med Rehabil Int - Volume 5 Issue 1 - 2018 ISSN : 2471-0377 | www.austinpublishing group.com Meiner et al. () All rights are reserved
Citation: Schwartz I, Gartsman I, Adler B, Friedlander Y, Manor O, Levine H, et al. High Prevalence of Cardiovascular Comorbidity and Chronic Pain in Polio Survivors as Compared to the General Population. Phys Med Rehabil Int. 2018; 5(1): 1138. 
Table 1: Demographic data of polio patients as compared to the general Israeli population.

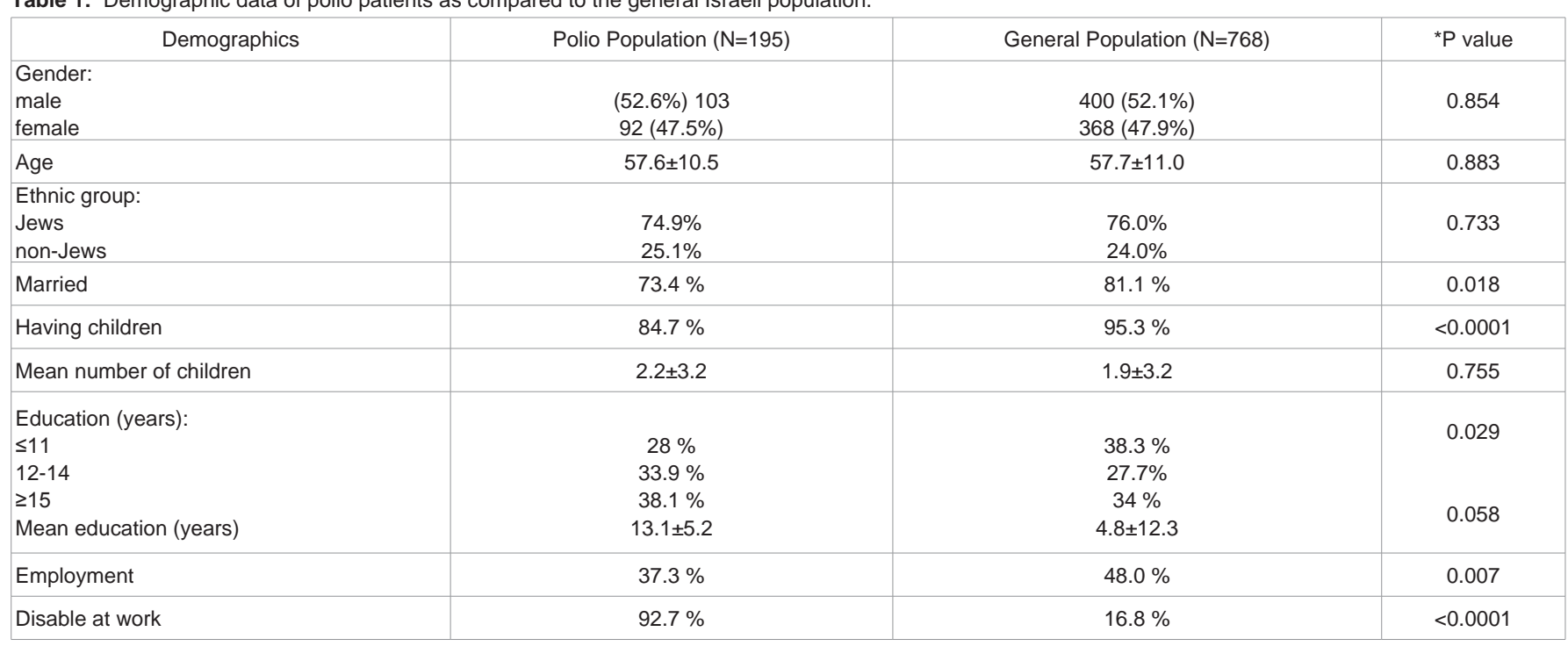

* $\mathrm{P}$ value for comparison between polio patients and general population.

in polio survivors and to developing programs aimed at their prevention.

\section{Methods}

\section{Participants}

This case-control study included 195 polio patients attending the post-polio clinic in PM\&R department of the Hadassah Mount Scopus Medical Center in Jerusalem. The study was approved by the ethics committee of Hadassah Medical center. The post-polio clinic in Hadassah Mount Scopus Medical Center was founded in 1997 and serves as a regional center for the polio population in Jerusalem and the surrounding areas, a region home to 800,000 inhabitants. Each patient is evaluated once every 6 months by a senior physiatrist with specialized expertise in polio and the post-polio syndrome. The evaluation entails a medical, neurological and functional examination. Following the evaluation, a physiatrist makes recommendations such as physical rehabilitation, specialized equipment and adaptations in home or work environments. All 209 patients of the post-polio clinic between 2010 until 2012 were asked to participate in this study; 195 patients accepted and 14 either refused or were unavailable. Each participant was interviewed by a rehabilitation physician experienced in the care of polio patients. The one hour interview usually took place in the PPS clinic in the hospital (92\%), in some cases in the patient's home $(5 \%)$ while $3 \%$ of the patients were interviewed by phone.

The control group was a random stratified sample from the international health survey which was conducted by the Israel Central Bureau of Statistic in 2003/2004 [11]. The health survey samples were divided into 28 strata by sex, (male-female) religion (Jews-non Jews) and age (45-49,50-54,55-59,60-64,65-69,70-74,75+), while from each stratum a random sample of controls was selected. The number controls selected was 4 times the number of polio cases in each stratum, with one exception; there were insufficient numbers of male non-Jews aged 45-49 and therefore for this stratum all available controls were selected. The control group sample included 768 controls with distribution of age, sex and religion comparable to the polio group.
Table 2: Functional status of polio population versus general Israeli population.

\begin{tabular}{|c|c|c|c|}
\hline Activities of Daily Living (ADL) & $\begin{array}{l}\text { Polio } \\
\mathrm{N}=195\end{array}$ & $\begin{array}{l}\text { General Population } \\
\qquad N=768\end{array}$ & ${ }^{\star} \mathrm{P}$ value \\
\hline Walking outdoors, (\%) & & & $<0.00001$ \\
\hline can & 13 & 76 & \\
\hline can with assistive devices & 65 & 7 & \\
\hline can't & 22 & 17 & \\
\hline Walking indoors, (\%) & & & $<0.00001$ \\
\hline can & 20 & 80 & \\
\hline can with assistive devices & 61 & 14 & \\
\hline can't & 19 & 6 & \\
\hline Washing, (\%) & & & $<0.00001$ \\
\hline can & 38 & 93 & \\
\hline can but difficult & 43 & 3 & \\
\hline can't & 19 & 4 & \\
\hline Dressing, (\%) & & & $<0.00001$ \\
\hline can & 42 & 93 & \\
\hline can but difficult & 41 & 3 & \\
\hline can't & 17 & 4 & \\
\hline
\end{tabular}

*P value for comparison between polio patients and general population

\section{Survey questionnaire}

The demographic, medical, social, and functional data of the polio patients was evaluated through the use of a questionnaire designed to correlate with the relevant sections of the National Health Surveys conducted by the Israel Central Bureau of Statistic in 2003/2004 [11]. The demographic data collected included current age, age of polio infection, gender, ethnic origin and place of birth. The socioeconomic data surveyed marital status, education and occupational status. Comorbidity data according to patient's report and family doctor diagnosis (ischemic or other heart diseases, stroke, high blood pressure, diabetes mellitus, chronic back or neck pain) was obtained from a comorbidity questionnaire, while level of difficulties in performing activities of daily living (ADLs) was obtained via a self- 
Table 3: Percentage of cardiovascular comorbidities, risk factors and chronic pain of polio survivors as compared to the general population.

\begin{tabular}{|c|c|c|c|}
\hline Morbidity & Polio N=195 & General Population N=768 & ${ }^{*} \mathrm{P}$ value \\
\hline \multicolumn{4}{|c|}{ Cardiovascular comorbidities and risk factors } \\
\hline MI, Heart disease or stroke & $40.5 \%$ & $18.2 \%$ & $<0.0001$ \\
\hline Hypertension & $44.3 \%$ & $33.8 \%$ & 0.006 \\
\hline Diabetes & $26.8 \%$ & $13.8 \%$ & $<0.0001$ \\
\hline BMI & $27.8 \pm 5.8$ & $27.1 \pm 4.5$ & 0.121 \\
\hline Obese $(\mathrm{BMI}>30)$ & $26.9 \%$ & $24.1 \%$ & 0.241 \\
\hline Active physically & $36.3 \%$ & $50.7 \%$ & 0.0004 \\
\hline $\begin{array}{c}\text { Smoking } \\
\text { Now } \\
\text { Past } \\
\text { Never }\end{array}$ & $\begin{array}{l}23.4 \% \\
23.4 \% \\
53.2 \% \\
\end{array}$ & $\begin{array}{l}23.9 \% \\
27.4 \% \\
48.7 \%\end{array}$ & 0.47 \\
\hline \multicolumn{4}{|l|}{ Chronic pain } \\
\hline Joint pain & $10.8 \%$ & $13.7 \%$ & 0.285 \\
\hline Back pain & $65.8 \%$ & $32.2 \%$ & $<0.0001$ \\
\hline Headache & $45.9 \%$ & $22.6 \%$ & $<0.0001$ \\
\hline Other pain & $58.8 \%$ & $13.6 \%$ & $<0.0001$ \\
\hline All Chronic Pain & $87.2 \%$ & $46.6 \%$ & $<0.0001$ \\
\hline
\end{tabular}

MI-Myocardial Infarct.

${ }^{*} \mathrm{P}$ value for comparison between polio patients and general population.

report questionnaire.

\section{Statistical methods}

Descriptive statistics were calculated for the continuous variables (mean, standard deviation and range) and differences between the two populations evaluated by t-test. Percentages and rates were calculated for the categorical variables and differences were assessed by chi square test. Logistic analysis was used to determine how well vascular risk factors and demographics predicted cardiovascular diseases and chronic pain Differences in the logistic function between polio survivors and general population were evaluated by adding interaction to the logistic regression. $\mathrm{P}<0.05$ was considered as significant.

\section{Results}

Demographic data of polio survivors in Jerusalem as compared to age and gender matched general population in Israel

The summary of the demographic data from the 195 (out of 269) polio patients who agreed to participate in the study, is presented in Table 1. The mean age of the polio survivors was $57.6 \pm 10.5$ (33-84); $52.6 \%$ men and $47.4 \%$ women. The group included 145 (74.9\%) Jews and $48(25.1 \%)$ Arabs, most of whom were Muslims. The percentage of those married was similar in both populations. Only $84.7 \%$ of the polio survivors had children as compared to $95.3 \%$ in the general population $(\mathrm{P}<0.0001)$, whereas the total number of children per family was not different between the two groups. Polio survivors were better educated than the general population with slightly higher mean years of education $(\mathrm{p}=0.058)$. According to our results, $37.1 \%$ of polio survivors were still employed as compared to $52.4 \%$ in the general population ( $\mathrm{p}=0.007$ ), with more than $90 \%$ of those employed reporting some disability at their work place. Nearly $50 \%$ of our polio population acquired the disease around their first birthday, almost $20 \%$ had involvement of 3 or more limbs, $50 \%$ suffered from scoliosis and $26 \%$ had respiratory disturbances. Almost $70 \%$ of the polio survivors required some orthoses for mobilization (data not shown). Regarding functional status, polio survivors were significantly less independent in their ambulatory capacity in and out of doors and in their ADL functions (Table 2).

\section{Cardiovascular comorbidities and chronic pain among polio survivors as compared to age and gender matched controls}

We found that both male and female polio survivors had significantly higher percentage of cardiovascular comorbidities including myocardial infarction, ischemic heart disease, stroke, hypertension and diabetes as compared to the general population (Table 3). For instance, the incidence of myocardial infarction was significantly higher among polio survivors as compared to the general population $(13.5 \%$ vs $5.8 \%, 0.0002)$. Within the polio group, the percentage of men with cardiovascular morbidities was significantly higher than that of women ( $49.5 \%$ vs $30.4 \%, \mathrm{p}=0.007$ ). A similar, yet expected, discrepancy was found between men and women in the general population $(21 \%$ vs $15.2 \%, \mathrm{p}=0.04)$. Other cardiovascular risk factors including the mean BMI, the percentage of people who were overweight and smoking rates (current and past) were found to be similar in the two groups. Polio survivors were found to be less physically active than their counterparts in the general population with only $36.3 \%$ of polio survivors engaged in any physical activity in the 30 days prior to testing as compared to $50.7 \%$ in the general Israeli population ( $\mathrm{p}=0.0004)$. They also reported a higher percentage of back pain, headache and other chronic pain, with the overall rate of pain in the polio population being $87.2 \%$ as compared to $46.6 \%$ in the control group $(\mathrm{P}<0.0001)$. Unlike the general population, men and women with polio reported similar rates of chronic pain $(87.4 \%$ vs $87.0 \%, \mathrm{p}=0.93$ ), while in the general population women suffered from significantly higher rates $(54.3 \%$ vs $39.5 \%, \mathrm{p}=0.0004)$. 


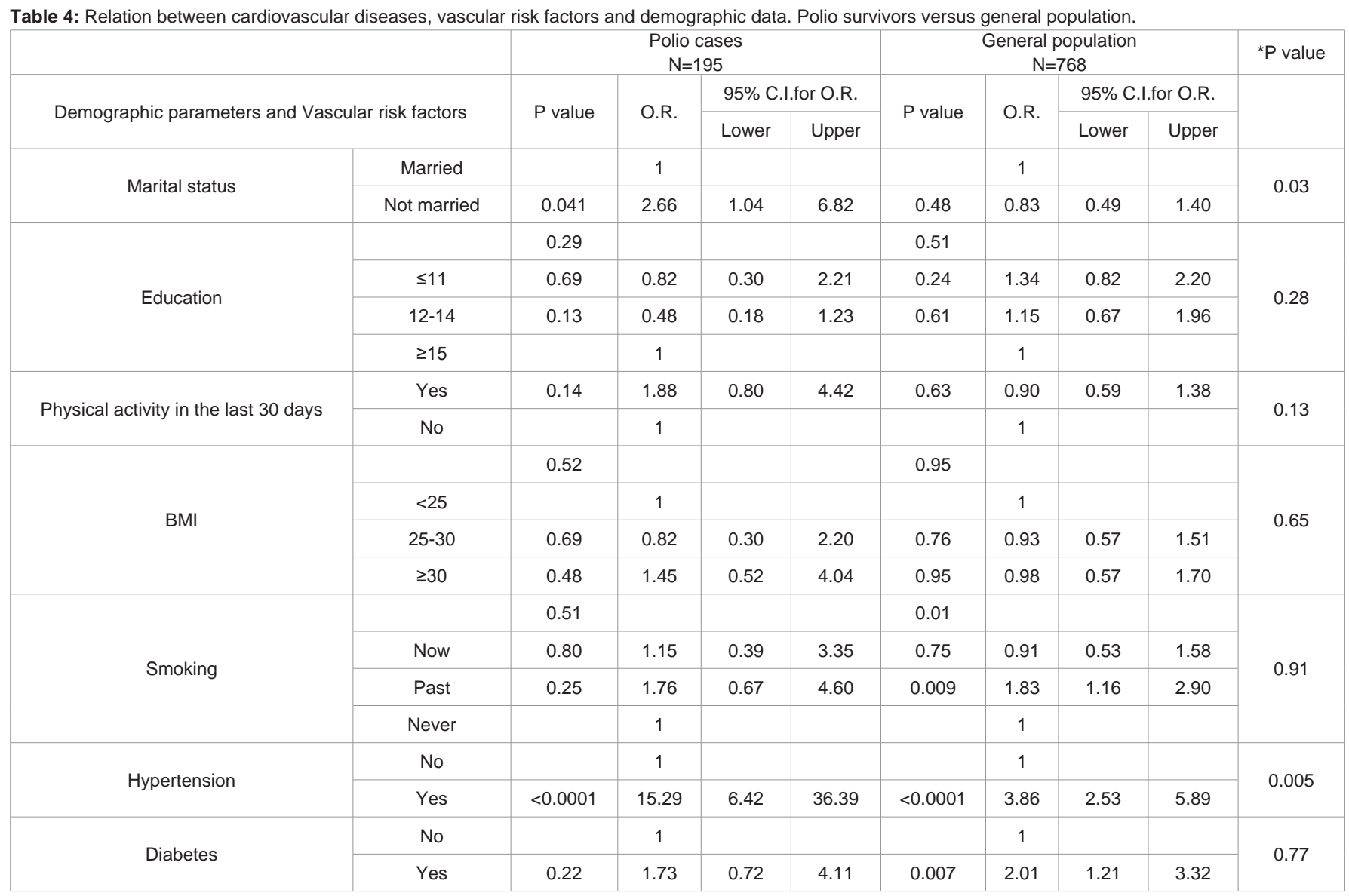

*P value for comparison between polio patients and general population.

Polio survivors utilized health services, such as visits to their family physician, nursing care and emergency rooms at significantly higher rates than the general Israeli population (data not shown).

The relationship between cardiovascular diseases or chronic pain and vascular risk factors and demographic data in polio survivors as compared to the general population

Using regression analysis, we compared the relationship between demographic data and history of stroke or heart disease, including myocardial infarct and vascular risk factors of the polio patients to the general population (Table 4). The vascular risk factors analyzed were hypertension, diabetes mellitus, smoking, obesity and physical activity. The demographic data included marital status and education. Surprisingly, in the polio population, single marital status correlated with a higher risk of cardiovascular diseases while in the general population such a correlation was not found. Hypertension correlated with higher risk of cardiovascular diseases in both populations but the magnitude of the influence was significantly higher in the polio population. On the other hand, history of smoking or diabetes was found to be related to higher risk of cardiovascular diseases only in the general population. Surprisingly, no association was found in either population between cardiovascular diseases and physical activity, BMI or educational level (Table 4).

No significant associations were found between any of the above parameters and chronic pain in polio survivors, or in comparison between polio patients and the general population (Table 5).

The relationship between cardiovascular diseases and chronic pain comorbidities and functional level in polio survivors as compared to the general population

In contrast to the general population, for whom medical comorbidities are directly and significantly related to level of functioning, for polio survivors neither cardiovascular diseases nor chronic pain were found to be related to physical activities or to basic ADLs (Table 6). The addition of hypertension and diabetes as variables to cardiovascular morbidity did not alter the results in either population (data not shown).

The relationship between cardiovascular disease and chronic pain and history and severity of polio in polio survivors

We evaluated the relationship between cardiovascular diseases and chronic pain and the following clinical and functional parameters: age of polio infection, mechanical ventilation due to respiratory involvement, kyphoscoliosis, number of limbs involved and current mobility (Table 7). No clinically significant correlation was found between any of these parameters with the risk of developing cardiovascular diseases or chronic pain.

\section{Discussion}

Our results indicate that polio survivors suffer from a higher prevalence of comorbidities such as cardiovascular conditions, 
Table 5: Relation between chronic pain, vascular risk factors and demographic data. Polio survivors versus general population.

\begin{tabular}{|c|c|c|c|c|c|c|c|c|c|c|}
\hline \multirow{3}{*}{\multicolumn{2}{|c|}{ Demographic parameters and Vascular risk factors }} & \multicolumn{4}{|c|}{$\begin{array}{c}\text { Polio cases } \\
\mathrm{N}=195\end{array}$} & \multicolumn{4}{|c|}{$\begin{array}{l}\text { General population } \\
\qquad N=768\end{array}$} & \multirow[t]{3}{*}{${ }^{*} \mathrm{P}$ value } \\
\hline & & \multirow{2}{*}{$P$ value } & \multirow{2}{*}{ O.R. } & \multicolumn{2}{|c|}{ 95\% C.I.for O.R. } & \multirow{2}{*}{$\mathrm{P}$ value } & \multirow{2}{*}{ O.R. } & \multicolumn{2}{|c|}{ 95\% C.I.for O.R. } & \\
\hline & & & & Lower & Upper & & & Lower & Upper & \\
\hline \multirow{2}{*}{ Marital status } & Married & & 1 & & & & 1 & & & \multirow{2}{*}{0.72} \\
\hline & Not married & 0.84 & 0.88 & 0.28 & 2.84 & 0.61 & 1.11 & 0.75 & 1.63 & \\
\hline \multirow{4}{*}{ Education } & & 0.19 & & & & .238 & & & & \multirow{4}{*}{0.42} \\
\hline & $\leq 11$ & 0.54 & 1.59 & 0.37 & 6.93 & 0.53 & 0.89 & 0.61 & 1.29 & \\
\hline & $12-14$ & 0.21 & 0.49 & 0.16 & 1.48 & 0.09 & 0.72 & 0.49 & 1.06 & \\
\hline & $\geq 15$ & & 1 & & & & 1 & & & \\
\hline \multirow{2}{*}{ Physical activity in the last 30 days } & Yes & 0.62 & 1.31 & 0.45 & 3.78 & 0.18 & 0.80 & 0.58 & 1.11 & \multirow{2}{*}{0.26} \\
\hline & No & & 1 & & & & 1 & & & \\
\hline \multirow{4}{*}{ BMI } & & 0.21 & & & & .104 & & & & \multirow{4}{*}{0.26} \\
\hline & $<25$ & & 1 & & & & 1 & & & \\
\hline & $25-30$ & 0.15 & 0.41 & 0.12 & 1.38 & 0.45 & 1.15 & 0.81 & 1.63 & \\
\hline & $\geq 30$ & 0.95 & 1.05 & 0.25 & 4.45 & 0.04 & 1.56 & 1.03 & 2.36 & \\
\hline \multirow{4}{*}{ Smoking } & & 0.40 & & & & 0.59 & & & & \multirow{4}{*}{0.40} \\
\hline & Now & 0.18 & 2.73 & 0.63 & 11.73 & 0.91 & 0.98 & 0.67 & 1.43 & \\
\hline & Past & 0.67 & 1.29 & 0.39 & 4.26 & 0.36 & 1.18 & 0.83 & 1.70 & \\
\hline & Never & & 1 & & & & 1 & & & \\
\hline \multirow{2}{*}{ Hypertension } & No & & 1 & & & & 1 & & & \multirow{2}{*}{0.77} \\
\hline & Yes & 0.40 & 1.60 & 0.54 & 4.76 & 0.0002 & 1.90 & 1.36 & 2.66 & \\
\hline \multirow{2}{*}{ Diabetes } & No & & 1 & & & & 1 & & & \multirow{2}{*}{0.21} \\
\hline & Yes & 0.30 & 0.56 & 0.18 & 1.70 & 0.42 & 1.20 & 0.77 & 1.89 & \\
\hline
\end{tabular}

*P value for comparison between polio patients and general population.

chronic pain, hypertension and diabetes when compared to the general population. Although the functional level of polio survivors was found to be diminished in comparison to the general population, a higher rate of cardiovascular disease or chronic pain did not correlate with level of disability for this group. Notwithstanding their severe disabilities, polio survivors succeeded in integrating well into society and achieving higher levels of education and sustained employment.

The demographic data of our sample was similar to other cohorts of polio survivors in Israel and around the world [12-19]. As in other studies, we found that polio survivors presented with greater incidences of comorbidities, including but not limited to cardiovascular problems (40.5\%), hypertension (44.3\%) and diabetes (26.8\%). Similarly, a previous Israeli study also reported higher percentages of comorbidities in polio survivors including hypertension (46.5\%), diabetes (17.8\%) and cardiac disease (12.9\%) [13]. In a study of 2,032 paralytic poliomyelitis patients in Taiwan, Kang and Lin found that patients with paralytic poliomyelitis had significantly higher prevalence of a wide range of diseases including cardiovascular diseases when compared to matched controls [10]. In a large study of stroke prevalence among 212 polio survivors in Taiwan, a higher prevalence of hypertension and stroke was found as compared to the general population [20]. Similar findings were reported from other studies in USA, Denmark and Norway [8,21-24].

Several important risk factors for cardiovascular diseases were investigated in our study including hypertension, diabetes, BMI, smoking and physical activity. As expected, hypertension and male gender were found to be significantly associated with higher rate of cardiovascular diseases both in groups. However, surprisingly, diabetes was associated with a higher rate of cardiovascular morbidity only in the general population. The BMI of our polio population as well as the presence of obesity (26.9\%) was not significantly higher than in general population. This finding is concurrent to previous studies conducted in the USA and Taiwan $[20,21]$ yet differs from a small study in Taiwan in which polio survivors had an unusual high prevalence of obesity [25]. In comparison with a study in 128 post-menopausal polio survivors women in USA [26], the mean BMI and the percentage of overweighed persons in our study was lower (mean BMI of 29.2 and $32 \%$ obesity [mean BMI $>30$ ] in the American study as compared to 27.8 and $26.9 \%$ in our study). These variations may reflect cultural and dietary differences between populations. We found that $46 \%$ of polio survivors were smokers or smoked in the past as compared to $52 \%$ in the general population, a rate higher than that reported in two American studies (20.4\% or 15\%) [21,26] but similar to a European study of 154 polio survivors in which $45 \%$ were smokers [27]. In our study, neither BMI nor smoking were found to be associated with higher cardiovascular morbidity rate in the polio population.

Regression analysis indicated that, with the exception of hypertension, the only variable that significantly predicted the rate of cardiovascular diseases in polio survivors was marital status. Polio survivors who were unmarried had a significantly increased risk of 
Table 6: Relation between cardiovascular diseases and chronic pain and functional status. Polio survivors versus general population.

\begin{tabular}{|c|c|c|c|c|c|c|c|c|c|c|c|c|c|}
\hline & & \multicolumn{6}{|c|}{ Cardiovascular Diseases } & \multicolumn{6}{|c|}{ Chronic Pain } \\
\hline & & \multicolumn{3}{|c|}{ Polio } & \multicolumn{3}{|c|}{ General } & \multicolumn{3}{|c|}{ Polio } & \multicolumn{3}{|c|}{ General } \\
\hline & & No & Yes & $\mathrm{P}$ & No & Yes & $\mathrm{P}$ & No & Yes & $\mathrm{P}$ & No & Yes & $\mathrm{P}$ \\
\hline \multirow{3}{*}{ walk out house } & can & 18 & 7 & 0.37 & 491 & 85 & $<0.01$ & 4 & 21 & 0.69 & 368 & 208 & $<0.01$ \\
\hline & can with help & 73 & 53 & & 45 & 12 & & 17 & 109 & & 16 & 41 & \\
\hline & can't & 24 & 19 & & 91 & 43 & & 4 & 39 & & 26 & 108 & \\
\hline \multirow{3}{*}{ walk in house } & can & 26 & 12 & 0.27 & 520 & 93 & $<0.01$ & 6 & 32 & 0.62 & 381 & 232 & $<0.01$ \\
\hline & can with help & 71 & 49 & & 77 & 29 & & 16 & 104 & & 19 & 87 & \\
\hline & can't & 18 & 18 & & 30 & 18 & & 3 & 33 & & 10 & 38 & \\
\hline \multirow{2}{*}{ wash } & can & 91 & 67 & 0.35 & 600 & 128 & 0.04 & 22 & 136 & 0.58 & 405 & 323 & $<0.01$ \\
\hline & can't & 24 & 12 & & 23 & 11 & & 3 & 33 & & 4 & 30 & \\
\hline \multirow[t]{2}{*}{ dress } & can & 92 & 69 & 0.24 & 600 & 128 & 0.15 & 22 & 139 & 0.58 & 405 & 323 & $<0.01$ \\
\hline & can't & 23 & 10 & & 22 & 9 & & 3 & 30 & & 4 & 27 & \\
\hline Total & & 115 & 79 & & 622 & 137 & & 25 & 169 & & 409 & 350 & \\
\hline
\end{tabular}

cardiovascular diseases yielding an adjusted odds ratio of 2.66 (95\% confidence interval, 1.04-6.82, $\mathrm{P}=0.041$ ). As mentioned above, the marital rate of polio survivors in our and in other studies was high but lower than the general population. One possible explanation is the general effect of marriage on health. In a large Israeli study, a protective effect of marriage was found for many conditions including hypertension [28], a finding in accordance with the protective effect of marriage on general health [29]. The protective effect of marriage on health may be explained by a more balanced life style and the mutual support of the spouse.

How can the higher rate of cardiovascular morbidities and cardiovascular risk factors among polio survivors be explained? The often noted justification found in the literature is that patients with paralytic poliomyelitis are less active physically and their life style more sedentary than the general population, therefore predisposing them to increased risk of cardiovascular complications. Previous studies have shown that polio survivors have declining cardiopulmonary function as compared to the general population, a factor which may increase their risk for cardiovascular diseases [30]. In our study as well, polio survivors were found to be less active than the general population with only $36.3 \%$ reporting regular exercise as compared to $50 \%$ of the general population. However, in regression analysis, we did not find a correlation between physical activity in the month prior and the prevalence of cardiovascular diseases among polio survivors. In addition, no correlation was found between the prevalence of cardiovascular diseases and the severity of polio sequelae or level of ambulation in polio survivors, measured by the usage of assistive devices or being confined to a wheelchair. Moreover, no correlation was found between parameters of primary polio infection such as age of onset or respiratory or skeletal involvement and the rate of cardiovascular morbidities. Therefore, our results cannot explain the higher rate of cardiovascular morbidities found in our polio population by the severity of motor disability and low level of physical activity. More studies are needed in order to answer this question.

Another major finding in our study was the high rate of chronic pain in our polio population. Muscle and joint pain assessment are part of the acceptable clinical scale for follow up of PPS severity
Table 7: Relation between history and severity of polio and cardiovascular disease and chronic pain.

\begin{tabular}{|c|c|c|c|c|c|c|c|}
\hline \multirow{2}{*}{\multicolumn{2}{|c|}{ Polio characteristics }} & \multicolumn{3}{|c|}{$\begin{array}{c}\text { Cardiovascular } \\
\text { disease }\end{array}$} & \multicolumn{3}{|c|}{ Chronic pain } \\
\hline & & No & Yes & $P$ value & No & Yes & $P$ value \\
\hline \multirow{3}{*}{ Age of polio onset } & $<1 \mathrm{Y}$ & 62 & 34 & \multirow{3}{*}{0.12} & 14 & 82 & \multirow{3}{*}{0.78} \\
\hline & $1-3 Y$ & 41 & 29 & & 8 & 62 & \\
\hline & $>3 Y$ & 12 & 16 & & 3 & 25 & \\
\hline \multirow{2}{*}{ Respiratory } & Yes & 23 & 25 & \multirow{2}{*}{0.12} & 2 & 46 & \multirow{2}{*}{0.28} \\
\hline & No & 84 & 54 & & 23 & 115 & \\
\hline \multirow{2}{*}{ Scoliosis } & Yes & 54 & 41 & \multirow{2}{*}{0.52} & 9 & 86 & \multirow{2}{*}{0.20} \\
\hline & No & 59 & 37 & & 16 & 80 & \\
\hline \multirow{2}{*}{$\begin{array}{l}\text { Number involved } \\
\text { limbs }\end{array}$} & 1 & 63 & 41 & \multirow{2}{*}{0.66} & 10 & 94 & \multirow{2}{*}{0.03} \\
\hline & $2<$ & 43 & 33 & & 13 & 63 & \\
\hline \multirow{2}{*}{ Using Aids } & Yes & 60 & 38 & \multirow{2}{*}{0.56} & 14 & 85 & \multirow{2}{*}{0.67} \\
\hline & No & 54 & 42 & & 11 & 85 & \\
\hline \multirow{2}{*}{ Using Wheelchair } & Yes & 48 & 21 & \multirow{2}{*}{0.02} & 10 & 60 & \multirow{2}{*}{0.66} \\
\hline & No & 65 & 59 & & 15 & 109 & \\
\hline \multirow{2}{*}{ Wheelchair bound } & Yes & 24 & 14 & \multirow{2}{*}{0.59} & 4 & 35 & \multirow{2}{*}{0.79} \\
\hline & No & 90 & 66 & & 21 & 135 & \\
\hline \multirow{3}{*}{ Motorized Wheelchair } & Yes & 19 & 9 & \multirow{2}{*}{0.31} & 3 & 25 & \multirow{2}{*}{0.96} \\
\hline & No & 95 & 71 & & 22 & 145 & \\
\hline & Total & 115 & 80 & & 25 & 168 & \\
\hline
\end{tabular}

$[31,32]$. Several explanations were suggested for this high degree of pain among polio survivors, the main being the phenomenon of overuse. In addition, previous studies indicated that female gender and lower extremity weakness were key factors in predicting the presence of muscle and joint pain in the polio population [33]. However, in our study, we did not find that female gender correlated with chronic pain in our polio population, whereas in the general population women reported significantly higher rates of pain. In previous studies it was reported that polio survivors performing at higher intensity levels were more likely to have moderate to severe pain and more mobility difficulties [34]. In our study, however, no 
demographic or clinical parameters were correlated with higher degrees of pain and no correlation was found between pain and any of the disease characteristics including age of onset, limb involvement and usage of assistive devices or wheelchairs. Of interest in our findings, in contrast to the case in the general population, that pain was not correlated with higher BMI in the polio group. Similar to our findings, in a study of 126 polio survivors, BMI was not found to be correlated with muscle pain and not significantly with joint pain [33]. The authors of that study concluded that the exact role of obesity in pain in polio survivors requires further study.

In our study, only in the general population there was a significant relationship between functional level and the occurrence of either cardiovascular diseases or chronic pain. This finding could be explained by the fact that the functional limitations of polio survivors were affected previously by their polio sequelae and were not significantly worsened by the additive effects of other comorbidities. On the other hand, in the general population, the presence of comorbidities such as cardiovascular diseases or chronic pain had significantly deleterious effect on their previously higher functional level.

Our study had several limitations, the number of polio patients was relatively small and came from the same polio clinic and residential area and therefore may not be representative of all polio survivors in Israel. Furthermore, polio patients who attend the postpolio clinic are likely to be more severely afflicted and therefore our sample may not be representative of the general post-polio population. Moreover, this study was based on questionnaires rather than on medical records, cholesterol levels or blood test results, which were unavailable to us.

\section{Conclusions}

Long-standing poliomyelitis has a deleterious impact on the general health and functional level of polio survivors. We found higher rate of cardiovascular comorbidity as well as chronic pain among polio survivors yet no correlation between these comorbidities and severity of motor disability, reduced physical activity or impaired functional levels. Our findings emphasize the need for early screening for vascular risk factors among polio survivors and promote the need for preventive and educational health programs in order to forestall further deterioration in this population.

\section{Acknowledgements}

This research was supported by the 2011 award of the Post-Polio Health International (PHI) Organization St. Louis, Missouri, USA and the Lawrence and Anita Miller Los Angeles Jewish federation rehabilitation research Fund.

\section{References}

1. Gonzalez H, Olsson T, Borg K. Management of postpolio syndrome. Lancet Neurol. 2010; 9: 634-642.

2. Halstaed LS. A brief history of postpolio syndrome in the United States. Arch Phys Med Rehabil. 2011; 92: 1344-1349.

3. McNalley TE, Yorkston KM, Jensen MP, Truitt AR, Schomer KG, Baylor C, et al. Review of secondary health conditions in postpolio syndrome: prevalence and effects of aging. Am J Phys Med Rehabil. 2015; 94: 139-145.

4. Schwartz TA. The Epidemiology of Polio in Israel. Dyonon Pub.Ltd. 2008.
5. Adler E, Eliakim C, Magora A. Poliomyelitis in Jerusalem in 1953. Acta Medica Orientalia. 1955; 14: 147-163.

6. Slater PE, Handsher R. Polio in Israel. Nature. 1989; 338: 110.

7. Polio survivors' report - selected characteristics. National Insurance Institution, Research and Planning Administration, Jerusalem, 2008.

8. Wekre LL, Stanghelle JK, Lobben B, Oéyhaugen S. The Norwegian Polio Study 1994: a nation-wide survey of problems in long-standing poliomyelitis. Spinal Cord. 1994; 36: 280-284.

9. Nielsen N, Rostgaard K, Askgaard D, Skinhøj P, Aaby P. Lifelong morbidity among Danes with poliomyelitis. Arch Phys Med Rehabil. 2004; 85: 385-391.

10. Kang JH, Lin HC. Comorbidity profile of poliomyelitis survivors in a Chinese population: a population-based study. J Neurol. 2011; 258: 1026-1033.

11. http://www.cbs.gov.il/www/briut/health0304.pdf

12. Jacob $\mathrm{T}$, Donchin $\mathrm{M}$, Levental $\mathrm{A}$. Late health problems among post polio survivors in Israel. Harefuah. 1995; 128: 477-481.

13. Jacob T, Shapira A. Quality of life and health conditions reported from two post-polio clinics in Israel. J Rehabil Med. 2010; 42: 377-393.

14. Zeilig G, Weingarden $\mathrm{H}$, Shemes $\mathrm{Y}$, Herman $\mathrm{A}$, Zeweker $\mathrm{M}$, et al. Functiona and environmental factors affecting work status in individuals with longstanding poliomyelitis. J Spinal Cord Med. 2012; 35: 68-69.

15. Farbu E1, Gilhus NE. Poliomyelitis: long-time consequences for social life. Acta Neurol Scand. 1997; 96: 353-358

16. Farbu E, Rekand T, Aarli JA, Gilhus NE. Polio survivors - well educated and hard working. J Neurol. 2001; 248: 500-505.

17. Rekand T, Kõrv J, Farbu E, Roose M, Gilhus NE, Langeland N, et al. Long term outcome after poliomyelitis in different health and social conditions. J Epidemiol Community Health. 2003; 57: 368-372.

18. Nollet F, Ivanyi B, Beelen A, De Haan RJ, Lankhorst GJ, De Visser M. Perceived health in a population based sample of victims of the 1956 polio epidemic in the Netherlands. J Neurol Neurosurg Psychiatry. 2002; 73: 695700.

19. Bang H, Suh JH, Lee SY, Kim K, Yang EJ, Jung SH, et al. Post-polio syndrome and risk factors in Korean polio survivors: a baseline survey by telephone interview. Ann Rehabil Med. 2014; 38: 637-647.

20. Wu C-H, Liou T-H, Chen H-H, Sun T-Y, Chen K-H, Chang K-H. Stroke risk in poliomyelitis survivors: a nationwide population-based study. Arch Phys Med Rehabil. 2012; 93: 2184-2188.

21. Gawne AC, Wells KR, Wilson KS. Cardiac risk factors in polio survivors. Arch Phys Med Rehabil. 2003; 84: 694-696.

22. Farbu E, Rekand T, Gilhus NE. Post-polio syndrome and total health status in a prospective hospital study. Eur J Neurol. 2003; 10: 407-413.

23. Farbu E1, Gilhus NE. Former poliomyelitis as a health and socioeconomic factor. A paired sibling study. J Neurol. 2002; 249: 404-409.

24. Lonnberg F. Late onset polio sequelae in Denmark. Results of a nationwide survey of 3,607 polio survivors. Scand J Rehabil Med, 1993; 28: 1-32.

25. Chang KH1, Lai CH, Chen SC, Hsiao WT, Liou TH, Lee CM. Body composition assessment in Taiwanese individuals with poliomyelitis. Arch Phys Med Rehabil. 2011; 92: 1092-1097.

26. Kalpakjian CZ, Riley BB, Quint EH, Tate DG. Hormone replacement therapy and health behavior in postmenopausal polio survivors. Maturitas. 2004; 48: 398-410.

27. Ragonese P, Fierro B, Salemi G, Randisi G, Buffa D, D'Amelio M, et al Prevalence and risk factors of post-polio syndrome in a cohort of polio survivors. J Neurol Sci. 2005; 236: 31-35.

28. Jaffe DH, Manor O, Eisenbach Z, Neumark YD. The protective effect of marriage on mortality in a dynamic society. Ann Epidemiol. 2007; 17: 540547.

29. Rendall MS, Weden MM, Favreault MM, Waldron $\mathrm{H}$. The protective effect of 
marriage for survival: a review and update. Demography. 2011; 48: 481-506.

30. Bargieri JV, Quadros AA, Pereira RD, Oliveira AJ, Lazaretti-Castro M, Silva AC. Basal metabolic rate and body composition in patients with post-polio syndrome. Ann Nutr Metab. 2008; 53: 199-204.

31. Kalpakjian CZ, Toussaint LL, Klipp DA, Forchheimer MB. Development and factor analysis of an index of post-polio sequelae. Disabil Rehabil. 2005; 27 1225-1233.

32. Brogårdh $\mathrm{C}$, Lexell $\mathrm{J}$, Lundgren-Nilsson $\mathrm{A}$. Construct validity of a new rating scale for self-reported impairments in persons with late effects of polio. PM\&R. 2013; 5: 176-181
33. Vasiliadis HM, Collet JP, Shapiro S, Venturini A, Trojan DA. Predictive factors and correlates for pain in postpoliomyelitis syndrome patients. Arch Phys Med Rehabil. 2002; 83: 1109-1115.

34. Klein MG, Keenan MA, Esquenazi A, Costello R, Polansky M. Musculoskeleta pain in polio survivors and strength-matched controls. Arch Phys Med Rehabil. 2004; 85: 1679-1683.
Phys Med Rehabil Int - Volume 5 Issue 1 - 2018

ISSN : 2471-0377 | www.austinpublishinggroup.com

Meiner et al. (C) All rights are reserved
Citation: Schwartz I, Gartsman I, Adler B, Friedlander Y, Manor O, Levine H, et al. High Prevalence of Cardiovascular Comorbidity and Chronic Pain in Polio Survivors as Compared to the General Population. Phys Med Rehabil Int. 2018; 5(1): 1138 